\title{
Moderator Roles of Optimism and Weight Control on the Impact of Playing Exergames on Happiness: The Perspective of Social Cognitive Theory Using a Randomized Controlled Trial
}

\author{
Huynh Van Nguyen, PhD, Han-Chung Huang, PhD, May-Kuen Wong, MD, PhD, \\ Ya-Hui Yang, MS, Tzu-Ling Huang, BS, and Ching-I Teng, PhD ${ }^{1,5,6}$
}

\begin{abstract}
Objective: The literature on exergames has examined their impact on user-perceived psychological health (i.e., user-perceived happiness), but little is known about whether such an impact depends on user characteristics. Therefore, this study used the perspective of social cognitive theory (SCT) to identify potential moderators (i.e., whether the user is optimistic or attempting to control his or her weight) of the impact of playing exergames on user-perceived happiness.

Materials and Methods: This large-scale randomized controlled trial recruited 337 college students (of whom $57.3 \%$ were female and all were aged $20-40$ years) as participants. The participants were randomly assigned to the intervention group or the control group. Participants in the intervention group were asked to use an Xbox 360 to play 1 of 10 exergame programs for 30 minutes once a week for 2 weeks. Participants in the control group were not required to do so. Repeated-measures ANOVA was used for the analyses.

Results: The analytical results indicate that playing exergames helped to maintain happiness levels and prevented them from decreasing. The maintained happiness was more prominent among participants who were trying to control their weight, but did not differ between participants who were highly optimistic and those who were less optimistic. This study is the first using SCT to explain the contingent effect of playing exergames on user happiness.

Conclusions: Exergames can maintain happiness among users, and such maintenance can be the strongest among users who are trying to control their weight.
\end{abstract}

Keywords: Exergame, Randomized controlled trial, Happiness, Optimism, Weight control

\section{Introduction}

$\mathbf{E}$ XERCISE IS WIDELY recognized as an effective means of promoting health. Elements of exercise and gaming are combined in exergames, ${ }^{1}$ and thus, exergames should be examples of games for health. Exergames are widespread around the world, as evidenced by the fact that one videogame device (Xbox 360) has sold 85 million units. ${ }^{2}$ Exergaming may effectively trigger behavior change. ${ }^{3}$ Moreover, college-aged individuals enjoy playing games, partly because they experienced enhanced happiness. Hence, research is needed to examine the sources of happiness in playing exergames, because such research may enhance happiness and attract college-aged individuals to frequently play exergames, thus benefiting their health.

The literature has examined the benefits of playing exergames, including greater physical activity levels, ${ }^{4}$ enjoyment, ${ }^{5}$ self-efficacy, ${ }^{6}$ positive mood change, ${ }^{7}$ enhancement in physical

\footnotetext{
${ }^{1}$ Graduate Institute of Business and Management, Chang Gung University, Taoyuan, Taiwan.

${ }^{2}$ Department of Rehabilitation, Chang Gung Memorial Hospital, Taoyuan, Taiwan.

${ }^{3}$ Institute of Health Policy and Management, National Taiwan University, Taipei, Taiwan.

${ }^{4}$ Department of Health Care Management, Chang Gung University, Taoyuan, Taiwan.

${ }^{5}$ Department of Rehabilitation, Chang Gung Memorial Hospital, Linkou, Taiwan.

${ }^{6}$ Department of Business and Management, Ming Chi University of Technology, New Taipei City, Taiwan.
} 
and cognitive function, ${ }^{8}$ improved physical fitness in the elderly, ${ }^{9}$ greater energy expenditure, ${ }^{10}$ the enhanced intention to engage in other forms of exercise, ${ }^{11}$ and physical activity of moderate intensity. ${ }^{12}$ Overall, literature has illustrated the health-related impacts of playing exergames. ${ }^{1,7-12}$

However, no study has yet investigated whether user characteristics (such as whether the user is optimistic or trying to control his or her weight) moderate the impact of playing exergames on happiness, indicating a research gap. Research filling this gap can clarify the contingent impact of playing exergames. Moreover, the results may provide evidence supporting the benefits of playing exergames, thus fostering customer loyalty, which is critical for managers in various fields. $^{13}$

A theory can provide a systematic explanation of a phenomenon and guide the selection of study variables, motivating us to search for a theory. To find an appropriate theoretical framework, we started from the features of happiness. Happiness can be the expected outcome of conducting a gratifying behavior (i.e., playing exergames). Conducting a behavior and expecting the outcomes are core elements of social cognitive theory (SCT). ${ }^{14}$ This theory, thus, should be applicable to our study.

SCT posits that human behavior is governed by personal, behavioral, and environmental determinants. ${ }^{14}$ When exergame users are confident in their abilities, they would expect to achieve favorable outcomes. Belief in achieving favorable outcomes is the essence of optimism ${ }^{15}$ (or the tendency to positively interpret daily information), motivating us to include optimism in the framework. According to the SCT, ${ }^{14}$ environmental determinants also regulate behavior. Hence, environmental determinants, such as how others view the user's appearance, may affect the user's behavior, thus motivating the users to attempt to control their weight by playing exergames. Often, those who are attempting to control their weight tend to exercise. Playing exergames resembles doing conventional exercise, supporting our inclusion of user weight control in the framework.

Therefore, the purpose of this study is to examine whether the impact of playing exergames on happiness depends on user characteristics (i.e., whether a user is optimistic and attempting to control his or her weight). This study makes several contributions to the literature. First, Ho et al. ${ }^{7}$ examined the relationship between positive mood experiences and game enjoyment among exergame users. Our study is novel in identifying and examining contingency when users perceive positive moods. Such contingency extends the current knowledge on exergaming.

Second, Lee et al. ${ }^{1}$ found that playing exergames could enhance positive moods among users. Our study is novel in examining whether such enhancement may be stronger for individuals possessing certain characteristics.

Third, Street et al. ${ }^{3}$ conducted a systematic review to summarize the impact of playing exergames on exercise behaviors and changes in weight indicators. Our study is new in focusing on whether a user is attempting to control weight.

Fourth, Laffan et al. ${ }^{16}$ investigated the relationship between videogame characteristics, videogame engagement, and general happiness among videogame users. Our study is new in examining the characteristics of users, rather than the characteristics of games. Moreover, our study is new in examining the link between characteristics and happiness in exergames, instead of games in general.

\section{Exergames and development of hypotheses}

Exergames require gamers to use physical movement to control gaming avatars to respond to gaming requirements and contexts. ${ }^{17}$ That is, playing exergames may achieve physical and psychological outcomes that are similar to those of doing other exercise.

Playing exergames has been shown to benefit individuals with specific health problems, such as those who sought to lose weight. Such play has increased self-efficacy among overweight and obese children, ${ }^{18}$ and improved gait and balance for poststroke patients. ${ }^{19}$ In addition, playing exergames provides pleasurable experiences, such as immersion and flow. ${ }^{20}$ These benefits and experiences may explain users' repeated play. ${ }^{13,21,22}$ Moreover, playing exergames typically creates happiness. ${ }^{23}$ Such findings indicate that playing exergames is an important source of happiness among users.

Positive impacts of playing exergames on user psychology include users' positive mood, ${ }^{5,24}$ motivation to further play exergames and do exercise, ${ }^{9}$ and positive health behavior change. ${ }^{24}$ That is, prior literature has agreed on the positive contribution of playing exergames on user happiness, motivating us to hypothesize a positive relationship between playing exergames and happiness.

\section{H1: Playing exergames is positively related to player's happiness}

Highly optimistic individuals tend to positively interpret daily information. ${ }^{15}$ Optimism enables individuals to adaptively cope with stress and leads them to focus on factors helping them to expect favorable outcomes. ${ }^{15}$ Adaptively coping with stress can be achieved by enhancing individuals' belief in their ability to perform an action/task (i.e., selfefficacy). According to SCT, ${ }^{14}$ self-efficacy and outcome expectations predict behavioral engagement. Therefore, exergame users who are highly optimistic are likely to be confident in their ability to positively cope with stress and achieve favorable gaming outcomes, which should thus make them feel happy. Hence, playing exergames could enhance happiness among those who are highly optimistic.

However, users who are less optimistic are less likely to positively interpret the outcome of playing exergames. The lack of a positive interpretation may lead individuals to evaluate playing exergames as a mix of pros and cons, offsetting the positive impact of playing exergames on enhanced happiness. Thus, we hypothesize:

\section{H2: Playing exergames is positively related to happiness for players who are more optimistic, but not positive to happiness for players who are less optimistic}

Often, individuals who aim to control their weight have the motivation to maintain or lose more weight. ${ }^{26}$ SCT posits that individuals' expectations result in behavioral engagement. ${ }^{14}$ Applied to the present research context, individuals who are attempting to control their weight would likely be motivated to expend their energy to better control their weight. Playing exergames is a form of energy expenditure, and thus can help control weight. Thus, playing exergames likely enhances happiness among those who are attempting to control their weight. 
However, those who are not attempting to control their weight may not place an emphasis on energy expenditure. As energy expenditure associated with playing exergames is not their focus, playing exergames may not markedly enhance their happiness. Thus, we hypothesized:

\section{H3: Playing exergames is positively related to happiness for players who are attempting to control their weight, but not positive to happiness for players who are not}

Figure 1 illustrates the research framework and the study hypotheses. All hypotheses are positively framed, including the main effect $(\mathrm{H} 1)$ and the moderating effects $(\mathrm{H} 2$ and $\mathrm{H} 3)$.

\section{Materials and Methods}

\section{Sample, data collection process, and trial design}

This study was a large-scale randomized control trial with two parallel groups. Most research team members were blind to the participants' assignments, as suggested by the methodological literature. ${ }^{27}$ The ethical aspects of the study were approved by the Institutional Review Board of Chang Gung Memorial Hospital (103-6878C).

We chose to focus on college-aged individuals, because they enjoy playing games. ${ }^{28}$ Among games, playing exergames could benefit their health. ${ }^{18}$ Hence, research is needed to examine how they could obtain happiness when playing exergames.

Participants were recruited through invitations posted on bulletin boards, online forums, and social networking sites, and through fliers that were distributed to students from February to May 2015. This study excluded individuals who reported that they had certain-defined diseases. A written informed consent process was implemented.

In total, 337 eligible participants were randomly assigned to the intervention group $(n=170)$ or the control group $(n=167)$. One participant in the intervention group did not continue with the intervention and another lost contact, leading to a total of 168 in the intervention group. All 167 participants in the control group were retained.

Immediately after the written informed consent process, participants were randomly assigned. Those who were assigned to the intervention group were directed to a laboratory equipped with Xbox 360 hardware and the Your Shape: Fitness Evolved exergaming software. These participants were asked to play the exergame for 30 minutes once a week for 2 weeks.

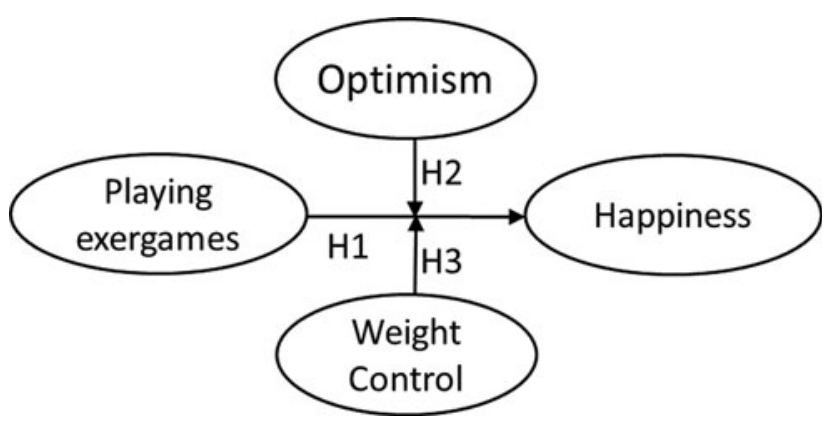

FIG. 1. Research framework.
The participants in the intervention group were randomly assigned to play 1 of 10 programs each time: African Rhythms, Wall Breaker, Hip-Hop, Yoga, Cardio, Cardio Boxing, Glutes, Health, Jump Rope, and Sports Preparation. Participants in the control group were not required to play the exergames. These participants completed the written informed consent process, completed a questionnaire, and came back the following week to complete another questionnaire. The moderators (i.e., optimism and weight control) were assessed before 2 weeks of participation in this study. The outcome variable, happiness, was assessed before and after the 2 weeks.

\section{Measurement, psychometric properties, and statistical analysis}

The items assessing user happiness were adapted from Lyubomirsky and Lepper. ${ }^{29}$ The four items were as follows: "In general, I am very happy," "Compared to most of my peers, I consider myself happy," "Regardless of what is going on, I enjoy my life," and "Generally, I am not very happy" (reverse coded). The maximum score of the scale was seven. The response option ranged from 1 (strongly disagree) to 7 (strongly agree). The four items assessing happiness had Cronbach's $\alpha$ values of 0.86 , indicating sufficient reliability.

The three items measuring optimism came from Richins. ${ }^{30}$ They were as follows: "Generally, I am optimistic," "Generally, I am encouraged," and "Generally, I am hopeful." The maximum score of the scale was four. The response option ranged from 1 (strongly disagree) to 4 (strongly agree). The items measuring optimism had Cronbach's $\alpha$ values of 0.83 . At the beginning of our study, we collected participants' responses on optimism and whether they were attempting weight control. The study constructs had correlations ranging from -0.01 to 0.46. Such correlations showed the minimum likelihood of common method variance.

This study adopted repeated-measures ANOVA to test the hypotheses. The dependent variable is happiness. The withinsubject factor is time (before and after the intervention/study). The between-subject factors are group (intervention vs. control), weight control (yes vs. no), and optimism (high vs. low).

\section{Results}

\section{Sample characteristics}

Among the participants in the intervention group, $53.0 \%$ were female and $93.5 \%$ were between 20 and 24 years old. Among the participants in the control group, $61.7 \%$ were female and $93.4 \%$ were between 20 and 24 years old. The participants in the two groups did not differ significantly in the proportion of male to female participants $(P=0.13)$. Moreover, the two groups did not differ significantly in age $(P=0.96)$.

\section{Hypothesis testing}

Table 1 lists the analytical results. Time did not increase happiness for all the participants $(F=1.06, P=0.30)$. Overall, the interaction of time and playing exergames had an impact on happiness $(F=6.63, P=0.01)$. This finding suggests that playing exergames helps maintain user happiness, supporting H1. This study found that playing exergames does not interact with optimism to impact happiness $(F=1.19$, $P=0.31$, not supporting $\mathrm{H} 2$. Playing exergames marginally 
Table 1. Repeated-Measures-ANOVA Results ON USER-PERCEIVED HAPPINESS

\begin{tabular}{lcc}
\hline & \multicolumn{2}{c}{ Happiness } \\
\cline { 2 - 3 } & $F$ & $\mathrm{P}$ \\
\hline Time & 1.06 & 0.30 \\
Time $\times$ playing exergames & 6.63 & 0.01 \\
Time $\times$ playing exergames $\times$ optimism & 1.19 & 0.31 \\
Time $\times$ playing exergames $\times$ weight control & 2.77 & 0.06 \\
\hline
\end{tabular}

interacted with whether the participant was attempting weight control $(F=2.77, P=0.06)$, supporting $\mathrm{H} 3$.

To further examine the potential interactions, we prepared Figure 2 to illustrate the main effects of playing exergames. We can observe that playing exergames helped maintain happiness levels and prevented them from decreasing. We further used four figures to illustrate the effect of playing exergames on users who had low levels of optimism (Fig. 3), high levels of optimism (Fig. 4), were not attempting weight control (Fig. 5), and were currently attempting weight control (Fig. 6). Statistically, optimism did not have a substantial moderating effect, while weight control did. Specifically, playing exergames markedly raised happiness levels for those who were currently attempting weight control, while the control group encountered a decrease in happiness over time, supporting the moderating role of weight control.

\section{Additional analysis}

We did not find a significant difference in gender composition, age, optimism at the beginning, proportion controlling weight, happiness at the beginning, and happiness at the end $(P \geq 0.11)$. Playing exergames likely maintained the happiness of the participants in the intervention group, while the happiness of those in the control group may have decreased. Table 2 summarizes the results.

\section{Discussion}

\section{Discussion on the hypothesis testing}

Analytical results supported $\mathrm{H} 1$ and $\mathrm{H} 3$. That is, playing exergames helps maintain user happiness (H1) and particu-

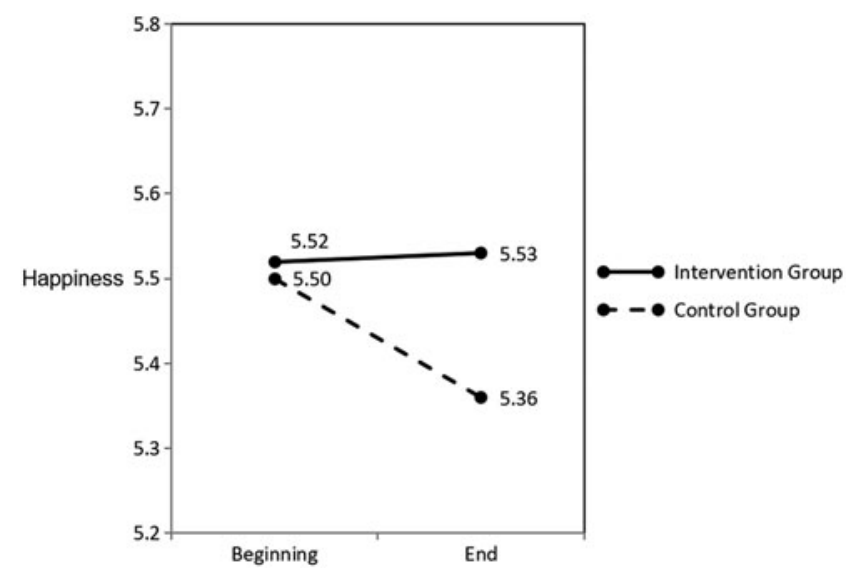

FIG. 2. Main effect of playing exergames.

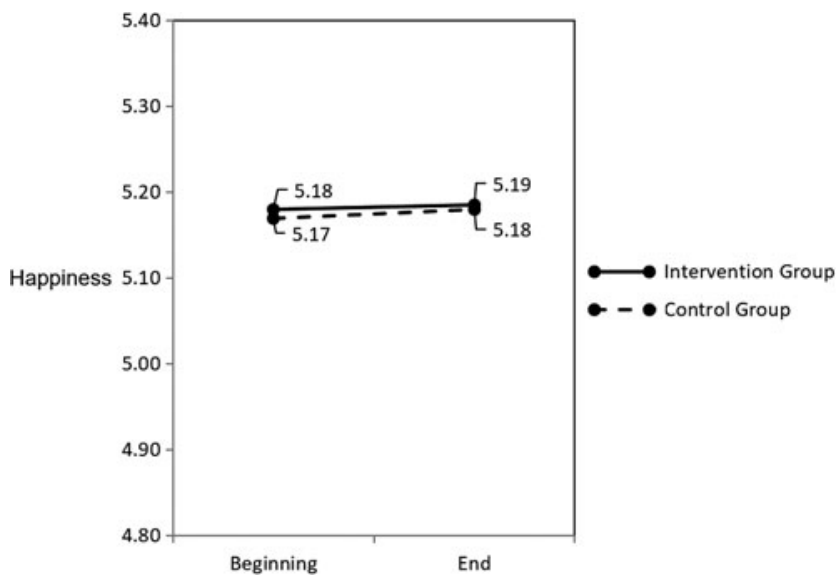

FIG. 3. Effect of playing exergames for less optimistic users.

larly helps maintain happiness among those who are attempting to control their weight (H3). However, $\mathrm{H} 2$ was not supported. The reason may be that highly optimistic individuals always take a positive perspective when interpreting their experiences. Therefore, they report uniformly high levels of happiness, reducing the magnitude of the interaction as predicted by $\mathrm{H} 2$.

\section{Main findings and contributions}

This study adopted a rigorous research design (i.e., a randomized controlled trial), indicating its strength. This study is the first to adopt the SCT to examine whether playing exergames positively contributes to user's psychological health (i.e., happiness). Overall, this study provided evidence supporting the positive contribution of exergames and identified two novel moderators (i.e., optimism and weight control).

This study contributes to exergaming literature by indicating that playing exergames provides a strong positive impact, particularly for participants who are attempting weight control and are highly optimistic. This study is the

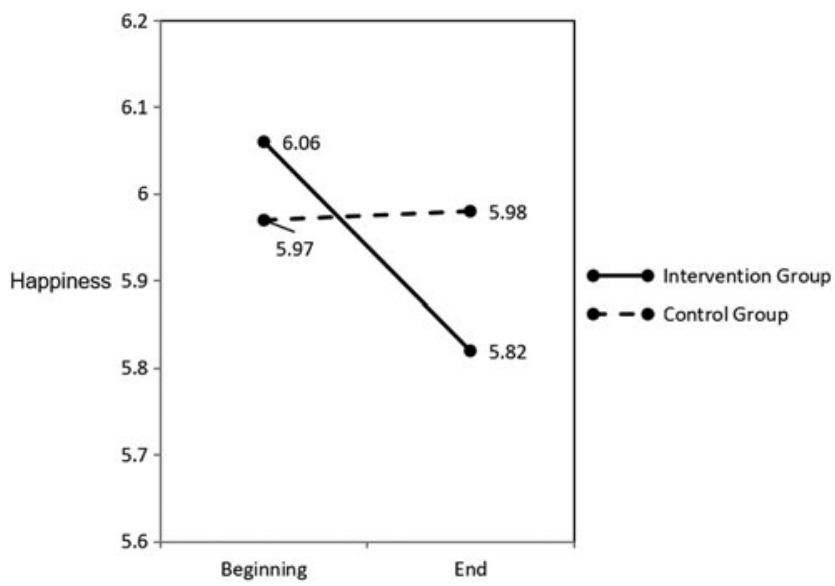

FIG. 4. Effect of playing exergames for highly optimistic users. 


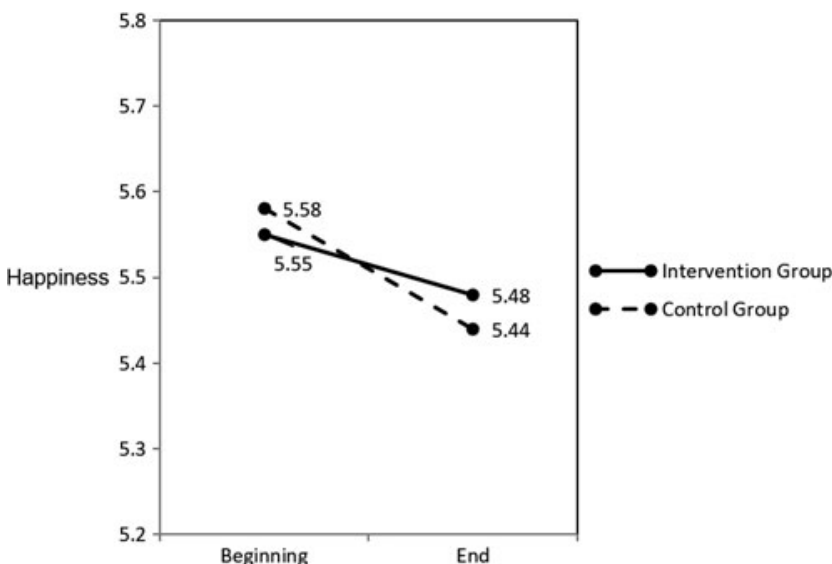

FIG. 5. Effect of playing exergames for users not attempting weight control.

first to examine the moderating role of these two factors (i.e., optimism and weight control).

\section{Theoretical implications}

We discussed the theoretical implications by comparing our findings with those in similar literature. Song et al. ${ }^{24}$ examined the impact of exergaming elements (i.e., competition) on users' intrinsic motivation, evaluation of the exergaming experience, and positive mood state. Our study is in line with theirs in verifying the impact of exergames on positive mood state. However, ours identifies a novel moderator of the impact (i.e., weight control). Such a moderator provides insights for exergame providers to effectively promote exergames.

Peña and $\mathrm{Kim}^{31}$ examined how avatar appearance (normal vs. obese) influences users' physical activity when playing exergames and found that users' physical activity increased when the avatar was at a normal appearance or obese. Individuals should regard appearance as very important, since the $\mathrm{SCT}^{14}$ posits that individual behavior could be regulated by environmental determinants, including how others view one's appearance. To keep a normal appearance, weight control may be one means for individuals. Therefore, future

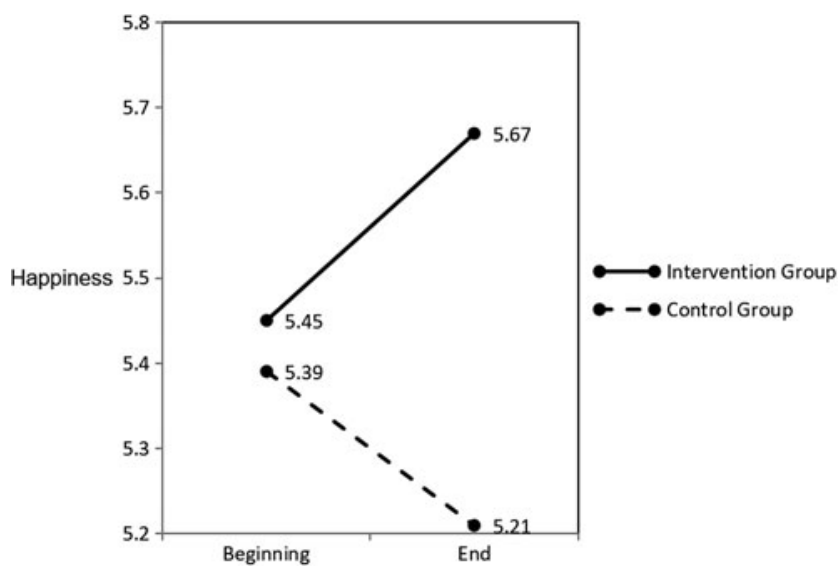

FIG. 6. Effect of playing exergames for users currently attempting weight control.
Table 2. Difference Between Intervention Group AND CONTROL Group

\begin{tabular}{lrr}
\hline Variable & Intervention & \multicolumn{1}{c}{ Control } \\
\hline $\begin{array}{l}\text { Gender composition } \\
\quad \text { proportion of males) }\end{array}$ & $0.46(0.50)$ & $0.38(0.49)$ \\
Age & $21.99(1.97)$ & $22.01(2.29)$ \\
Optimism in the beginning & $3.13(0.56)$ & $3.14(0.57)$ \\
Proportion of weight control & $0.26(0.44)$ & $0.25(0.44)$ \\
Happiness in the beginning & $5.52(0.91)$ & $5.50(0.88)$ \\
Happiness at the end & $5.53(0.97)$ & $5.36(0.94)$
\end{tabular}

None of their differences was significantly different from zero. Numbers were the mean values, while the numbers in the parentheses were the standard deviations.

works could explore how the two different factors, that is, normal appearance and weight control, interact with users' behavior in playing exergames.

Street et $\mathrm{al}^{3}{ }^{3}$ concluded that playing exergames was positively related to changes in exercise behavior, body weight, body fat mass, body fat percentage, and waist and hip circumference among heavy users of exergames. Our study has a unique focus on moderators of the impact on user happiness.

Recent exergaming literature found that playing exergames improved users' attitudes toward other forms of exercise, ${ }^{9}$ users' physical fitness, ${ }^{18}$ vigor, and happiness. ${ }^{24}$ Compared with those studies, the present study is innovative in identifying a novel moderator (i.e., controlling weight), in the impact of playing exergames on user happiness. Such an innovation has not been covered by previous studies, $9,18,24$ further demonstrating the present study's uniqueness and contributions.

\section{Practical implications}

Built on SCT, this study examines the impact of playing exergames on users' perceived happiness and the moderators of such an impact. This impact is more prominent for users who are attempting to control their weight. Thus, the findings of this study can be applicable or generalizable to other industries, such as Internet of Things companies in the fitness industry.

This study found that playing exergames can maintain happiness. This finding provides evidence for exergame providers' claims to their potential users that playing exergames can further enhance their positive mood. Exergame providers can include this information in their promotional activities and encourage potential users to try playing exergames. These trials by users would likely maintain their happiness.

This study found that playing exergames maintained happiness among users who were attempting to control their weight. Therefore, exergame providers could increase the options to indicate the spent calories and the equivalent minutes of doing other exercise. Such an option could be useful for users who are controlling their weight.

These findings also provide insights that benefit users. Specifically, users can frequently play exergames to maintain their happiness. Such happiness can be maintained particularly when users are attempting to control their weight. 


\section{Research limitations and future research direction}

Our sample comprised undergraduate and graduate students. This approach may restrain the generalizability to other age groups. Future studies could replicate this research in other age groups.

We asked participants to self-report whether they were controlling their weight. That is, we did not enforce some participants to control their weight. Future studies may explore whether weight control can be incorporated into the intervention, thus boosting the impact of playing exergames.

This study asked the participants to play exergames by appointment when it was convenient for them. However, this approach restrained us from controlling or examining the effect of playing at certain times of day. Future works may extend this study by examining this issue.

This study randomly assigned the participants in the intervention group to play 1 of the 10 programs each time they played. This approach should balance the effects of playing each program on happiness, and maximize the generalizability of the study findings to multiple exergame programs. However, this approach restrains us from knowing the most effective program for boosting happiness. Future works may explore how to design the most effective exergame program.

\section{Conclusion}

This study found that playing exergames helps maintain happiness among users. Moreover, this maintenance was more prominent among users who were attempting weight control. The findings of this study show that the impact of playing exergames is contingent on users' attempt to control their weight.

\section{Acknowledgment}

The authors thank Chang Gung Memorial Hospital, Taiwan, for financial support for this study (CMRPD3D0022).

\section{Author Disclosure Statement}

No competing financial interests exist.

\section{References}

1. Lee JE, Xiang P, Gao Z. Acute effect of active video games on older children's mood change. Comput Human Behav 2017; 70:97-103.

2. Statista. Video game console sales worldwide for products total lifespan as of June 2016 (in million units). 2017. www.statista.com/statistics/268966/total-number-ofgame-consoles-sold-worldwide-by-console-type (accessed August 17, 2017).

3. Street TD, Lacey SJ, Langdon RR. Gaming your way to health: A systematic review of exergaming programs to increase health and exercise behaviors in adults. Games Health J 2017; 6:136-146.

4. Golden D, Getchell N. Physical activity levels in children with and without autism spectrum disorder when playing active and sedentary Xbox Kinect videogames. Games Health J 2017; 6:97-103.

5. Lyons EJ. Cultivating engagement and enjoyment in exergames using feedback, challenge, and rewards. Games Health J 2015; 4:12-18.
6. Zeng N, Pope Z, Gao Z. Acute effect of virtual reality exercise bike games on college students' physiological and psychological outcomes. Cyberpsychol Behav Soc Netw 2017; 20:453-457.

7. Ho SS, Lwin MO, Sng JRH, et al. Escaping through exergames: Presence, enjoyment, and mood experience in predicting children's attitude toward exergames. Comput Human Behav 2017; 72:381-389.

8. Chao YY, Scherer YK, Montgomery CA. Effects of using Nintendo Wii (TM) exergames in older adults: A review of the literature. J Aging Health 2015; 27:379-402.

9. Larsen LH, Schou L, Lund HH, et al. The physical effect of exergames in healthy elderly-A systematic review. Games Health J 2013; 2:205-212.

10. Sween J, Wallington SF, Sheppard V, et al. The role of exergaming in improving physical activity: A review. J Phys Act Health 2014; 11:864-870.

11. Nguyen HV, Huang HC, Wong MK, et al. Double-edged sword: The effect of exergaming on other forms of exercise; a randomized controlled trial using the self-categorization theory. Comput Human Behav 2016; 62:590-593.

12. Verhoeven K, Abeele VV, Gers B, et al. Energy expenditure during Xbox Kinect play in early adolescents: The relationship with player mode and game enjoyment. Games Health J 2015; 4:444-451.

13. Li BJ, Lwin MO. Player see, player do: Testing an exergame motivation model based on the influence of the self avatar. Comput Human Behav 2016; 59:350-357.

14. Bandura A. Social Foundations of Thought and Action: A Social Cognitive Theory. Englewood: Prentice-Hall; 1986.

15. Scheier MF, Carver CS. Effects of optimism on psychological and physical well-being: Theoretical overview and empirical update. Cognit Ther Res 1992; 16:201-228.

16. Laffan DA, Greaney J, Barton H, et al. The relationships between the structural video game characteristics, video game engagement and happiness among individuals who play video games. Comput Human Behav 2016; 65:544549.

17. Huang HC, Wong MK, Lu J, et al. Can using exergames improve physical fitness? A 12-week randomized controlled trial. Comput Human Behav 2017; 70:310-316.

18. Staiano AE, Abraham AA, Calvert SL. The Wii club: Gaming for weight loss in overweight and obese youth. Games Health J 2012; 1:377-380.

19. Wüest S, Borghese NA, Pirovano M, et al. Usability and effects of an exergame-based balance training program. Games Health J 2014; 3:106-114.

20. Lee S, Kim W, Park T, et al. The psychological effects of playing exergames: A systematic review. Cyberpsychol Behav Soc Netw 2017; 20:513-532.

21. Bronner S, Pinsker R, Noah JA. Physiological and psychophysiological responses in experienced players while playing different dance exer-games. Comput Human Behav 2015; 51:34-41.

22. Peng W, Crouse J. Playing in parallel: The effects of multiplayer modes in active video game on motivation and physical exertion. Cyberpsychol Behav Soc Netw 2013; 16 : 423-427.

23. Huang HC, Wong MK, Yang YH, et al. Impact of playing exergames on mood states: A randomized controlled trial. Cyberpsychol Behav Soc Netw 2017; 20:246-250.

24. Song H, Kim J, Tenzek KE, et al. The effects of competition and competitiveness upon intrinsic motivation in exergames. Comput Human Behav 2013; 29:1702-1708. 
25. Kim SYS, Prestopnik N, Biocca FA. Body in the interactive game: How interface embodiment affects physical activity and health behavior change. Comput Human Behav 2014; 36:376-384.

26. Williams GC, Grow VM, Freedman ZR, Ryan RM, Deci EL. Motivational predictors of weight loss and weight-loss maintenance. J Pers Soc Psychol 1996; 70:115-126.

27. Schulz KF, Grimes DA. The Lancet: Handbook of Essential Concepts in Clinical Research. Edinburgh: Elsevier; 2006

28. Teng CI. Managing gamer relationships to enhance online gamer loyalty: The perspectives of social capital theory and self-perception theory. Comput Human Behav 2018; 79: 59-67.

29. Lyubomirsky S, Lepper HS. A measure of subjective happiness: Preliminary reliability and construct validation. Soc Indic Res 1999; 46:137-155.
30. Richins ML. Measuring emotions in the consumption experience. J Consum Res 1997; 24:127-146.

31. Peña J, Kim E. Increasing exergame physical activity through self and opponent avatar appearance. Comput Human Behav 2014; 41:262-267.

Address correspondence to: Ching-I Teng, PhD Graduate Institute of Business and Management Chang Gung University 259, Wenhua 1st Road, Gueishan Taoyuan 333 Taiwan

E-mail: chingit@mail.cgu.edu.tw 